\title{
ZUR FLEISCHVERSORGUNG KONSTANTINOPELS
}

Die Lebensmittelversorgung Konstantinopels wurde lange Zeit primär aus der Perspektive der Versorgung mit Brotgetreide untersucht. ${ }^{1}$ Erst in den letzten Jahren haben auch andere Aspekte die Aufmerksamkeit der Gelehrten auf sich gezogen: die Versorgung mit Frischgemüse, Fisch und Trinkwasser. ${ }^{2}$ Ausgespart blieb dagegen der hauptstädtische Fleischmarkt, ${ }^{3}$ obwohl sich in den Quellen genügend Hinweise darauf finden, daß frisches oder konserviertes Fleisch, obwohl kein Grundnahrungsmittel, ${ }^{4}$ für die Ernährung der hauptstädtischen Bevölkerung eine nicht zu unterschätzen-

${ }^{1}$ S. etwa J. L. Teall, The Grain Supply of the Byzantine Empire 330-1025. DOP 13 (1959) 87-139 mit der älteren Literatur; speziell für die Spätantike s. P. Herz, Studien zur römischen Wirtschaftsgesetzgebung. Die Lebensmittelversorgung (Historia Einzelschriften 55). Stuttgart 1988, 302-330; A. E. MüLLER, Getreide für Konstantinopel. Überlegungen zu Justinians Edikt XIII als Grundlage für Aussagen zur Einwohnerzahl Konstantinopels im 6. Jahrhundert. JÖB43(1993) 1-20; J. DurLIAT, L'approvisionnement de Constantinople, in: Constantinople and its Hinterland. Papers from the Twentyseventh Spring Symposium of Byzantine Studies, Oxford April 1993. Aldershot 1995, 19-33; P. Magdalino, The Grain Supply of Constantinople Ninth-Twelfth centuries, in: Constantinople and its Hinterland, 35-47; s. auch J. Koder, Gemüse in Byzanz. Die Versorgung Konstantinopels mit Frischgemüse im Lichte der Geoponika. Wien 1993, 99-108.

${ }^{2}$ S. Koder, Gemüse passim; ders., Fresh vegetables for the Capital, in: Constantinople and its Hinterland, 49-55; G. DaGron, Poissons, pêcheurs et poissonniers de Constantinople, ibid. 57-75; C. Mango, The Water Supply of Constantinople, ibid. 9-17.

${ }^{3}$ Kurz und nur auf die Spätantike beschränkt bleibt Herz, Lebensmittelversorgung 325-328; knapp auch M. F. Hendy, Studies in the Byzantine Monetary Economy c. 300-1450. Cambridge 1985, 562-565.

${ }^{4}$ Hendy, Monetary Economy 561 zählt Schweine- und Schaffleisch zu den Grundnahrungsmitteln, was für die große Mehrheit der Bevölkerung nicht zutrifft, s. Tealt, Grain Supply 98-99; E. Patlagean, Pauvreté économique et pauvreté sociale à Byzance $4^{\mathrm{e}}-7^{\mathrm{e}}$ siècles. Paris 1977, 38-41; A. Karpozilos - A. P. Kazhdan, ODB I 621-622 s. v. Diet; zu den Grundnahrungsmitteln vgl. ferner Albert von Aachen I 15 (283 F-G Bongars), der Getreide, Wein, Öl und Milchprodukte erwähnt. Zu einem möglichen Anstieg des Fleischverzehrs gegenüber der Spätantike s. A. Kazhdan, Two Notes on Byzantine Demography in the Eleventh and Twelfth Centuries. BF 8 (1982) 117-122. 
de Rolle gespielt hat. Das belegen nicht zuletzt die zwölf die Fleischversorgung betreffenden Erlasse des Eparchenbuchs, das unsere wichtigste Quelle für den hauptstädtischen Vieh- und Fleischmarkt darstellt. ${ }^{5}$ Allerdings wirft der Text des Eparchenbuchs eine Reihe von Problemen auf, die die Anzahl der mit der Fleischversorgung und Vermarktung beteiligten Korporationen betreffen, die Modalitäten des Handels und namentlich die Preisfestlegung sowie die Frage, ob das Eparchenbuch eine vollständige Sammlung aller für den Fleischmarkt relevanten Konstitutionen enthält. Darüber hinaus sollen die Herkunftsregionen des Schlachtviehs identifiziert und die Art und Weise untersucht werden, auf die das Vieh auf die konstantinopolitanischen Märkte gelangte. Schließlich gilt es, die Interessen des Staates zu klären und seine Maßnahmen sie durchzusetzen, zu beurteilen. All diesen Aspekten soll in der folgenden Untersuchung nachgegangen werden.

\section{Zum Problem der Korporationen und der von ihnen Bewirtschafteten Fleischsorten}

Die Kapitel 15 und 16 des Eparchenbuches, die sich mit dem Schlachtviehhandel und dem Fleischverkauf beschäftigen, sind gemäß ihren Überschriften zwei verschiedenen Korporationen gewidmet: den Metzgern

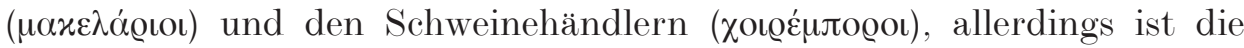

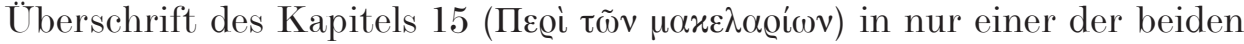
Handschriften nachträglich eingefügt worden. ${ }^{6}$ Diese Überschriften sind in gewisser Hinsicht irreführend. In den beiden Kapiteln werden zwei verschiedene Bereiche der Fleischversorgung behandelt, nämlich im Kapitel 15 der Markt für Schaf- und Lammfleisch und möglicherweise auch für Rindfleisch, ${ }^{7}$ während im Kapitel 16 ausschließlich der Schweinefleischmarkt behandelt wird; außerdem sind nicht zwei, sondern vier Korporationen mit jeweils sorgfältig abgegrenzten Zuständigkeiten involviert. ${ }^{8} \mathrm{Im}$ Kapitel 15 sind dies zunächst die $\mu \alpha x \varepsilon \lambda \alpha \dot{\varrho}$

${ }^{5}$ Eparchenbuch 15,1-16,6 (608-651 KoDER); dreizehn, wenn man die Versorgung mit konserviertem Fleisch mit einbezieht, s. Eparchenbuch 13,1 (563 Koder).

${ }^{6}$ Nämlich im Codex S, wobei eine schwarze Tinte verwendet wurde, s. den Kritischen Apparat zum Eparchenbuch 607 KodER; ansonsten verwendete der Schreiber von S für die Kapitelüberschriften rote Tinte, s. KodER, Eparchenbuch 52.

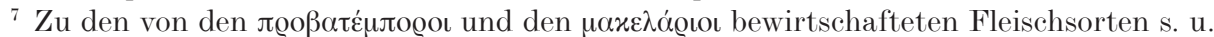

${ }^{8}$ Daß das Eparchenbuch nicht alle hauptstädtischen Korporationen unter einer jeweils gesonderten Rubrik abhandelt, wurde von der Forschung längst erkannt, s. S. VRYonIS JR., $\triangle$ HMOKPATIA and the Guilds in the Eleventh Century. DOP 17 (1963) 297 A. 26. 
dem Viehmarkt am Strategion, teils auch von den Erzeugern direkt ankauften, um sie sodann zu schlachten, zu zerlegen und den Verbrauchern

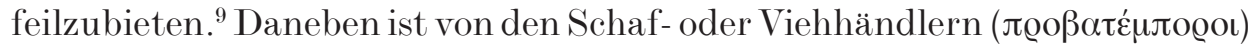
die Rede, die das Vieh von den Züchtern erwarben, um es auf dem Strategion an die Metzger weiterzuverkaufen. ${ }^{10}$ Mit der Schlachtung der Tiere und der Vermarktung des Fleisches hatten sie nichts zu tun, diese oblag den

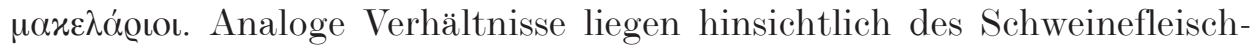

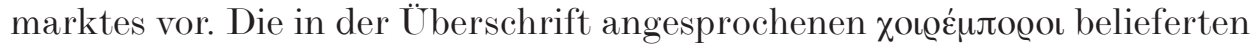
den Schweinemarkt am Tauros mit lebenden Tieren, wo sie von den Schwei-

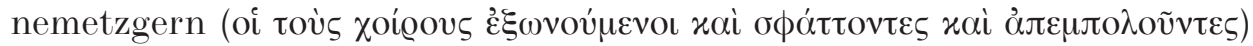
angekauft, geschlachtet und zum Verkauf angeboten wurden. ${ }^{11}$

Die Belieferung des Fleischmarktes durch mehrere, jeweils für eine Fleischsorte zuständige Korporationen entsprach einer aus der römischen Zeit überkommenen Tradition: im spätantiken Rom wurde der Fleischmarkt von drei verschiedenen Korporationen versorgt: den suarii (Schweinefleisch), den pecuarii (Schaf- und Ziegenfleisch) und den boarii (Rindfleisch). ${ }^{12}$ Ein Vergleich mit den für das mittelalterliche Konstantinopel überlieferten Verhältnissen fördert jedoch einige wichtige Unterschiede zu Tage. In den spätantiken Quellen wird nämlich nicht zwischen Händlern und Metzgern unterschieden, offenbar übten die suarii, boarii und pecuarii jeweils beide Tätigkeiten aus. ${ }^{13}$ Zudem wurde der stadtrömische Fleischmarkt - und hier vor allem der Schweinefleischmarkt - zu einem beträchtlichen Teil mit Steuertieren beliefert; ${ }^{14}$ Hinweise darauf fehlen für Konstantinopel, ebensowenig wissen wir irgendetwas über kaiserliche Fleischspenden an bedürftige Teile der hauptstädtischen Bevölkerung, wie sie für Rom bezeugt sind. ${ }^{15}$

Der signifikanteste Unterschied läßt sich hinsichtlich der von den einzelnen Korporationen bewirtschafteten Fleischsorten feststellen. Die $\chi 0$ -

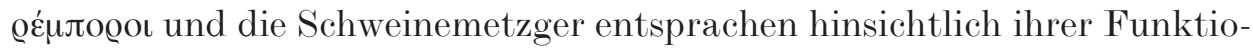

\footnotetext{
${ }^{9}$ Eparchenbuch 15,1-3 (607-620 KODER).

${ }^{10}$ Eparchenbuch 15,4-5 (621-628 KoDER).

11 Eparchenbuch 16,2-3 (635-643 KODER).

${ }^{12}$ Zum stadtrömischen Fleischmarkt in der Spätantike s. Herz, Lebensmittelversorgung 277-294, der sich ihrer Bedeutung und ihrer Prominenz in den Quellen entsprechend auf die suarii konzentriert und die boarii übergeht. Die drei Korporationen werden allerdings in einem Gesetz aus dem Jahre 452 gemeinsam erwähnt, s. Novellae Valentiniani 36 (153 Meyer - Mommsen).

13 S. CIL VI 1770,4-22; Codex Theodosianus 14,4,10 (782 Mommsen); Herz, Lebensmittelversorgung 279.

${ }^{14} \mathrm{Zu}$ den Funktionen der beiden Korporationen s. Herz, Lebensmittelversorgung 278 $279 ; 283$.

15 S. Codex Theodosianus 14,4,10 (782-783 Mommsen).
} 
nen den suarii, sie handelten ausschließlich mit Schweinen und Schweine-

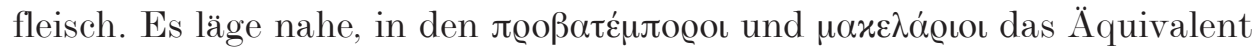

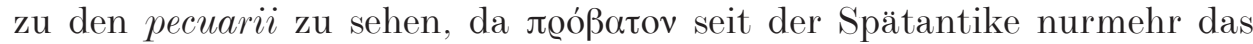
Schaf bezeichnete. ${ }^{16}$ Das wirft allerdings die Frage auf, wer den hauptstädtischen Markt mit Rindfleisch versorgte, denn für diese Fleischsorte ist weder im Eparchenbuch noch in einer anderen Quelle eine eigene Korporation überliefert. ${ }^{17}$

Verfehlt wäre es freilich, daraus die Schlußfolgerung zu ziehen, daß Rinder für die hauptstädtische Fleischversorgung als eine zu vernachlässigende Größe eingeschätzt werden können, es kann im Gegenteil als sicher gelten, daß Rindfleisch stets eine beachtliche Rolle gespielt hat. ${ }^{18}$ Demnach muß es in Analogie zu den Verhältnissen auf dem Schaf- und Schweinefleischmarkt sowohl Rindviehhändler als auch Rindviehmetzger gegeben haben, die aber im Eparchenbuch nicht nachweisbar sind. Dafür bieten sich zwei Erklärungsmöglichkeiten an: entweder wurde der Rindermarkt bei der Redaktion des Eparchenbuches vergessen oder übergangen ${ }^{19}$ oder aber

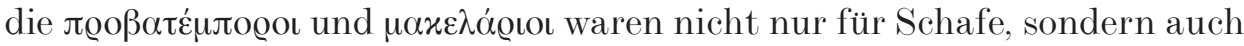
für Rinder zuständig. ${ }^{20}$ Wie die Festlegung der Höchstpreise für die von den $\mu \alpha x \varepsilon \lambda \alpha \dot{\varrho} \iota \circ$ anzukaufenden Tiere, von der noch die Rede sein wird, deutlich macht, scheidet die zweite Möglichkeit aus. Es hat also zwei Korporationen der Rinderhändler und Rindermetzger gegeben, die im Eparchenbuch nicht aufgeführt sind und die den Markt mit Rind- und Kalbfleisch versorgten. Das Fehlen dieser für die hauptstädtische Lebensmittelversorgung

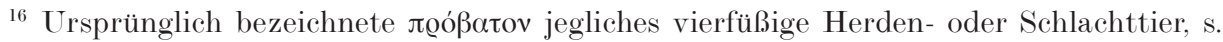

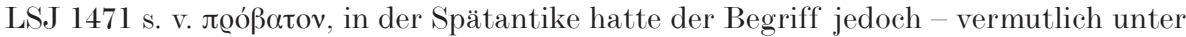
dem Einfluß der christlich- theologischen Literatur - die Bedeutung von „Schaf" angenommen, s. G. W. H. Lampe, A Patristic Greek Lexicon. Oxford 1961, 1139 s. v.

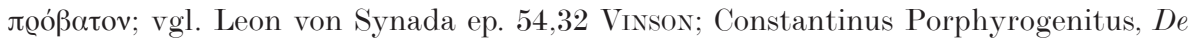
cerimoniis Appendix C 535 HaLdon.

17 Die Eparchenbuch 21,1-9 (736-782 Koder) behandelten Bothroi handelten mit Reitund Tragtieren, aber nicht mit Rindern.

${ }_{18} \mathrm{~S}$. dazu u.

19 Daß das Eparchenbuch kein vollständiges Verzeichnis sämtlicher hauptstädtischer Zünfte darstellt, wurde von verschiedenen Gelehrten betont, s. VRyonis, Guilds 296297; J. Koder, Überlegungen zu Aufbau und Entstehung des Eparchikon Biblion, in: Kathegetria. Essays presented to Joan Hussey for her 80th Birthday. Camberley 1988, 87; P. Speck, (Erlassenes) Gesetz oder ein weiteres Schulbuch. Überlegungen zur Entstehung des Eparchenbuches, in: Varia III (ПOIKIAA BYZANTINA 11) 304.

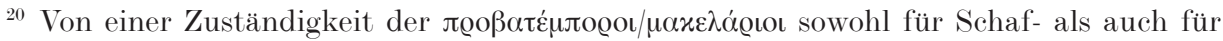

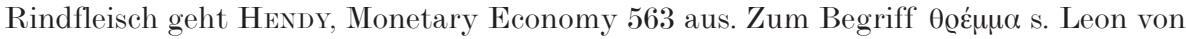
Synada ep. 54,34 Vinson, er wird dort für die Gesamtheit des nach Konstantinopel gesandten Viehs ohne Ansehen der Art benutzt, vgl. Leon von Synada ep. 54,31-32 Vinson. 
durchaus wichtigen Korporationen erweckt Zweifel daran, daß dem Entstehen des Eparchenbuchs ein einheitlicher, durchdachter Plan oder gar ein gesetzgeberischer Wille zugrunde lag. ${ }^{21}$

\section{Die verschiedenen Fleischsorten und ihre Bedeutung}

Die bloße Tatsache, daß eine Korporation im Eparchenbuch genannt wird, sagt also nichts über die Bedeutung der von ihr bewirtschafteten Fleischsorte für die Ernährung der konstantinopolitanischen Bevölkerung aus. In absoluten Zahlen gesehen, haben auf den hauptstädtischen Viehmärkten sicher mehr Schafe und Schweine als Rinder auf Käufer gewartet. ${ }^{22}$ Rückschlüsse auf das Mengenverhältnis der von den Schlachtern angebotenen Fleischsorten sind dennoch nicht ohne weiteres möglich; hier fällt nämlich buchstäblich ins Gewicht, daß ein Schlachtrind in etwa den siebenfachen Fleischertrag eines Schafes und den vierfachen eines Schweins erbrachte. ${ }^{23}$ Für den kaiserlichen Haushalt, für den wir in einem Appendix

${ }^{21}$ KoDER, Eparchenbuch 31-32 denkt an eine angesichts des bevorstehenden Todes Leons VI. (912) vorgenommene überhastete Endredaktion. Diese kann vielleicht ,inhaltliche Mängel, Inkonsequenzen in der Gliederung und Unausgewogenheiten“ (KoDER, Eparchenbuch 32) erklären, aber kaum das Fehlen wichtiger Korporationen, zumal mit der Sammlung der zu verarbeitenden Materialien laut Koder (Eparchenbuch 31, vgl. 21) spätestens 885/6(!) begonnen worden sein soll. An der von KODER, Eparchenbuch 21 postulierten Praxisorientiertheit des Eparchenbuchs, von der auch Vryonis, Guilds 297 grundsätzlich ausgeht, sind folglich Zweifel angebracht. Die These von Speck, Schulbuch 306, daß das Eparchenbuch letzlich nur eine krude Materialsammlung gewesen sei, hat einiges für sich, wenngleich ich mich der Ansicht dieses Gelehrten (a.a.O.), es handele sich um die „Grundlage für ein Schulbuch für den jungen Konstantin VII.“, nicht anschließen kann.

${ }^{22}$ Vgl. O. PickL, Der Viehhandel von Ungarn nach Oberitalien vom 14. bis zum 17. Jahrhundert, in: Internationaler Ochsenhandel (1350-1750). Akten des 7th International Economic History Congress Edinburgh 1978. Stuttgart 1979, 44; 67 A. 4 zu den Verhältnissen in Venedig, wo trotz der überragenden Bedeutung des Ochsenhandels für die Fleischversorgung in Stückzahlen betrachtet weit mehr Kleinvieh (1529: ca. 70.000) als Rinder und Kälber (ca. 14.000 bzw. 13.000) auf den Markt gelangten.

${ }^{23}$ S. die Tabelle bei B. KrüGER et al., Die Germanen. Geschichte und Kultur der germanischen Stämme in Mitteleuropa I. Berlin 1976, 442; die Zahlen sind zwar auf die germanische Viehzucht bezogen, dürften jedoch als Verhältnisangaben auch für die hier behandelte Periode Gültigkeit haben, zumal Krüger bei der Schätzung des Lebendgewichts der Schweine ausdrücklich Angaben aus dem Mittelalter heranzieht. Krüger nimmt für ein germanisches Schlachtrind maximal ein Gewicht von $300 \mathrm{~kg}$ Lebendgewicht an; laut I. N. Kiss, Die Bedeutung der ungarischen Viehzucht für Ungarn und Mitteleuropa vom 16. bis zum 18. Jahrhundert, in: Internationaler Ochsenhandel 107 rechnete man vor der Einführung der speziellen ungarischen Fleischrinderrasse im 14. Jahrhundert pro gemästetem Rind mit höchstens 3,7 Dz. 
des Zeremonienbuches genauere Angaben besitzen, wurden auf Feldzügen neben 300 Stück Geflügel 100 Milchlämmer, 500 Schafe und 50 Rinder als Schlachtvieh mitgeführt ${ }^{24}$ bei solchen Zahlen ist davon auszugehen, daß Lamm- und Schaffleisch mengenmäßig überwogen. Allgemeingültigkeit können diese Angaben jedoch nicht beanspruchen, da es sich hier um die kaiserliche Tafel handelt und Lamm- und Schaffleisch eine höhere Wertschätzung genoß als Rindfleisch. ${ }^{25}$ Kekaumenos (2. Hälfte 11. Jahrhundert) empfiehlt die Zucht von Schafen und Schweinen zum Verzehr an der eigenen Tafel, während Rinder nur als Zugtiere Erwähnung finden, ${ }^{26}$ doch vertritt er den Standpunkt des wohlhabenden Grundbesitzers, und seine Aussagen können nicht ohne weiteres verallgemeinert werden. Die Bedeutung des Rindes als Zugtier und Milchlieferant darf nicht darüber hinwegtäuschen, daß sein Fleisch auch im östlichen Mittelmeerraum immer verzehrt worden ist. ${ }^{27}$ So spielte es eine beachtliche Rolle für die Heeresversorgung, zumal bei den einfacheren Soldaten, ${ }^{28}$ und auch für Konstantinopel selbst besitzen wir Hinweise darauf, daß Rindfleisch konsumiert wurde. ${ }^{29}$

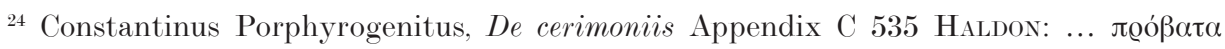

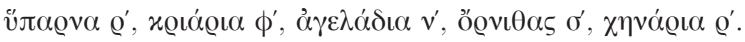

${ }^{25}$ Lammfleisch galt als saftig und leichtverdaulich, während Rindfleisch zwar als nahrhaft, aber auch als schwer verträglich beurteilt wurde, s. Symeon Seth, Syntagma de alimentorum facultatibus 20,10-15; 26,15-19 Langkavel; vgl. auch Рн. I. Kukules,

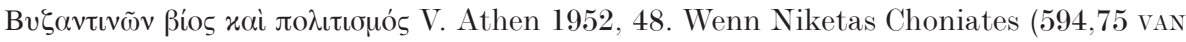
Dieten) die Lateiner verächtlich als „Rindfleischfresser“ abqualifiziert, ist das vor diesem Hintergrund zu sehen. Speziell zur kaiserlichen Tafel s. Liutprand von Cremona, Legatio 20 (MGH SS in usum scholarum 41, 186,16-18): ... haedum pinguem ...; schwerer als die abschätzigen Bemerkungen Liutprands über das fette Zicklein und die Art seiner Zubereitung wiegt die Tatsache, daß es sich um eine Delikatesse von der kaiserlichen Tafel handelte, von der der Kaiser zuvor selbst gespeist hatte; zu der Stelle s. auch T. Weber, Essen und Trinken im Konstantinopel des 10. Jahrhunderts, nach den Berichten Liutprands von Cremona, in: Liutprand von Cremona in Konstantinopel. Wien 1980, 90-91.

${ }^{26}$ Kekaumenos, Strategikon 88 (36,17-22 WAssiliewsky - JernstedT).

${ }^{27}$ Zum Rindfleischverzehr vgl. etwa Athenaios I 27 e (I 62,12 KAIBEL); Priscus fr. 11,2,2527 Blockley; Belege speziell für die Spätantike auch bei Patlagean, Pauvreté 41.

${ }^{28}$ S. den Appendix C des Zeremonienbuches (596 Haldon); Michael Attaleiates 113,11-12

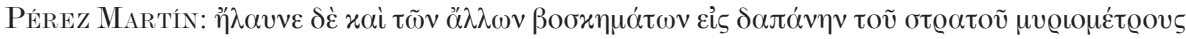

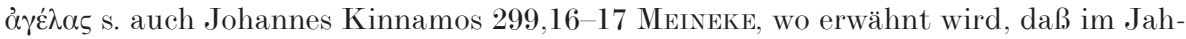
re 1176 für den großen Salğūqenfeldzug Manuels I. 3000 Rinder aus Thrakien herangetrieben wurden, wobei es sich allerdings zum Teil um Zugochsen gehandelt haben kann, vgl. Anna Komnena 7,11,6 (235,94-95 Reinsch - Kambylis).

${ }^{29}$ S. bes. Niketas Choniates 594,2-3 van Dietex; vgl. Leon von Synada, ep. 54,31-32 VINSON. 
Als preiswertere und in großen Mengen verfügbare Fleischsorte ${ }^{30}$ dürfte es speziell für die unteren Schichten der Bevölkerung von Bedeutung gewesen sein.

Schwierig ist es auch, die Bedeutung des Schweinefleischs zu beurteilen. In Italien und Sizilien besaß die Schweinezucht seit alters her einen besonders hohen Stellenwert, ${ }^{31}$ und noch im spätantiken Rom war Schweinefleisch die wichtigste Fleischsorte gewesen. ${ }^{32}$ Für die Fleischversorgung des östlichen Mittelmeerraums hingegen kann die Bedeutung des Schweins als Schlachtvieh insgesamt wahrscheinlich deutlich geringer veranschlagt werden. ${ }^{33}$ Allerdings müssen bezüglich der Schweinehaltung stärker als bei der Rinder- und Schafzucht regionale Unterschiede beachtet werden. Eine extensive Schweinehaltung wurde vom Vorhandensein größerer Eichenund Kastanienwälder stark begünstigt, die eher auf dem Balkan als in Kleinasien zu finden waren. ${ }^{34}$ So fehlen Schweine völlig in der Liste des bei Feldzügen im Osten für den kaiserlichen Tisch mitzuführenden Schlachtviehs. Nur Pökelfleisch wird verzeichnet, ${ }^{35}$ dies wird mit den besonderen Schwierigkeiten zusammenhängen, lebende Schweine unter den klimatischen und ökologischen Bedingungen des ost- und südostkleinasiatischen Kriegsschauplatzes über einen längeren Zeitraum mitzuführen. ${ }^{36}$ Bei Feldzügen in den europäischen Reichsteilen mag das anders gewesen sein, denn

${ }^{30}$ Zur Verfügbarkeit vgl. Johannes Kinnamos 299,16-17 Meineke; vgl. Villehardouin 444-445 (II 258,16-260,2 FARAL); 492 (II 306,3-10 FARAL).

$31 \mathrm{Zu}$ den Verhältnissen in Italien vgl. Polybios II 15,2-3 (I 140,27-141,3 BütTnERWoBst), der auf die Existenz großer Eichenwälder als Grundlage der Schweinezucht hinweist; zum sizilianischen Schweineexport s. Athenaios I 27 f (I 62,15 KAIBEL).

32 Die Quellen berichten von umfangreichen kaiserlichen Maßnahmen mit dem Ziel, die Preise niedrig zu halten und kostenlose Verteilungen zu ermöglichen, dazu ausführlich Herz, Lebensmittelversorgung 162-169; 171-172; 277-294.

33 Schweine fehlen in den spätantiken Katastern von Mytilene (s. IG XII.2, 76) und Thera (s. IG XII.3, 343; 346) völlig, während Schafe, Rinder und Ziegen aufgeführt sind; s. auch Themistios 15,186 c-d (I 271,6-15 Downey), wo nur Rinder und Schafe erwähnt werden; Schweinehaltung für die Gegend von Edessa ist im frühen 6. Jahrhundert durch Josua Stylites 38 (WRIGHT) bezeugt, sie stand aber in ihrer Bedeutung hinter der Rinder- und Kleinviehzucht zurück, s. Josua Stylites 38 (33,22-34,1 WRIGHT); vgl. 69 (67,9 WRIGHT); 75 (79,12 WRIGHT).

34 S. A. Kazhdan - J.W. Nesbitt, ODB III 1979-1980 s. v. Swine; vgl. o. A. 31. Die Wichtigkeit von Wäldern und feuchten Wiesen betont Columella 7,9,6 (202,33-37 RICHTER); $7,9,7$ (203,41,44 RiCHTER).

35 Constantinus Porphyrogenitus, De cerimoniis Appendix C 146 Haldon.

$36 \mathrm{Vgl}$. Hendy, Monetary Economy 564. Grundsätzlich dürfte sich hier der hohe Wasserbedarf der Tiere nachteilig ausgewirkt haben, s. Columella 7,10,6 (210,32-212,42 RICHTER). 
Schweine begegnen bei der Aufzählung des Viehs, das von Richard Löwenherz 1190 im Feldlager des zyprischen Usurpators Isaak Komnenos erbeutet wurde. ${ }^{37}$ Rückschlüsse auf den Schweinefleischkonsum in Konstantinopel sind anhand solcher Quellenbelege leider nicht möglich. Bezüglich der Schweinehaltung haben wir freilich einen Aspekt zu berücksichtigen, der für die Rinder- und Schafzucht nicht gegeben war, nämlich die Aufzucht von Schweinen durch städtische Privathaushalte, sei es zur Ergänzung der eigenen Ernährung, sei es, um sich vermittels des Verkaufs der Tiere eine (zusätzliche) Einnahmequelle zu erschließen. ${ }^{38}$ Für Konstantinopel wissen wir durch einen Brief des Johannes Tzetzes (Mitte 12. Jh.) von einem Angehörigen des hauptstädtischen Klerus, der in seiner Etagenwohnung Schweine hielt. ${ }^{39}$ Welchen Umfang die Selbstversorgung hatte und in wieweit sie den städtischen Schweinefleischmarkt beeinflußte, läßt sich nicht abschätzen, Verhältnisse wie die von Tzetzes beschriebenen brauchen aber in Konstantinopel durchaus keine Ausnahme gewesen zu sein. ${ }^{40}$ Grundsätzlich galt Schweinefleisch als eine wertvolle Fleischsorte, ${ }^{41}$ und manche Gelehrte sind sogar geneigt, dem Schweinefleischkonsum das Etikett des Tafelluxus anzuhängen. ${ }^{42}$ Anhand der wenigen Zeugnisse, die Preisvergleiche ermöglichen, läßt sich in der Tat feststellen, daß die Preise für Schweinefleisch über denen für Rind- und Hammelfleisch lagen; dennoch sind die Unterschiede nicht so kraß, daß man Schweinefleisch gleich zum Luxusnahrungsmittel stempeln kann. ${ }^{43}$

Wegen des höheren Preises gehe ich davon aus, daß Schweinefleisch in Konstantinopel in geringeren Mengen verzehrt wurde als Rind- und

${ }^{37}$ S. Ambroise, L'Estoire de la Guerre Sainte 1679 PARIs; Itinerarium Peregrinorum 2,33 (194,9 Stubis). Gleichfalls erwähnt werden Ochsen, Kühe, Ziegen, Schweine, Hammel, Schafe, Lämmer, Hähne, Hühner, Kapaune, s. Ambroise, L’Estoire de la Guerre Sainte 1679. 1681. 1683 PARIs; Itinerarium Peregrinorum 2,33 (194,7-9 StubBs).

38 Dieses Phänomen ist bereits für die Antike belegt, s. Athenaios 14,656 f (III 453,9-14 KaIBEL). Wir haben es hier nicht mit einer Zucht, sondern nur mit der Aufzucht und Mast preiswert gekaufter Ferkel zu tun, s. auch Columella 7,9,4 (202,23-24 Richter).

39 Johannes Tzetzes, ep. 18 (20,24-26 Pressel $=33,7-8$ Leone).

${ }^{40}$ Vgl. Eparchenbuch 16,4 (644-645 KODER).

${ }^{41}$ Zur Beurteilung des Schweinefleischs s. Symeon Seth, Syntagma de alimentorum facultatibus 119,16-22 Langkavel, der Zartheit, leichte Verdaulichkeit und Nährwert hervorhebt; s. auch Kukules 53-54.

${ }^{42}$ S. P. WIRTh, Lebenshaltungskosten in Konstantinopel im Jahre 1200 n. Chr. JÖB 44 (1994) 452; vgl. bereits Platon, Politeia II 373 c 4-6 Burnet.

${ }^{43}$ Vgl. zusammenfassend G. Ostrogonsky, Löhne und Preise in Byzanz, BZ 32 (1932) 327-333; nach dem Preisedikt Diokletians kostete Schweinefleisch pro Pfund zwischen 12 und 20 Denaren (4,1a. 5 Lauffer), während die Preise für Rind-, Hammel- und Ziegenfleisch bei acht Denaren lagen (4,2-3 Lauffer). 
Schaffleisch. Denkbar ist aber, daß vor allem nach der Eroberung Bulgariens im frühen 11. Jahrhundert das Angebot an Schweinefleisch zunahm, die Preise fielen und der Schweinefleischkonsum in der Hauptstadt zunahm, ohne daß sich weitergehende Aussagen begründen ließen.

Nicht erwähnt wird vom Eparchenbuch der Handel mit Geflügel und Wildbret. Mengenmäßig fiel das Geschäft mit diesen als Delikatessen geltenden Fleischsorten sicher nicht ins Gewicht. Der Verzehr von Wild und Wildgeflügel blieb abgesehen vom kaiserlichen Hof in erster Linie auf „Selbstversorger“ beschränkt, meist Angehörige der Oberschicht, die auf ihren Landgütern dem Jagdvergnügen frönten, daneben auch auf solche Bevorzugten, denen Wildbret als Geschenk übersandt wurde. ${ }^{44}$ Bei Zuchtund Hausgeflügel spielte die häusliche Selbstversorgung - namentlich bei Hühnern - sicher gleichfalls eine wichtige Rolle ${ }^{45}$, und man kann annehmen, daß auf diesem Sektor auch die Selbstvermarktung die Regel war, zumal es eines Metzgers nicht bedurfte. Besondere Wild- und Geflügelhändlergilden anzunehmen halte ich daher für unnötig, was allerdings die Existenz von einzelnen, spezialisierten Delikatessenhändlern nicht ausschließt.

\section{Die Herkunftsregionen des Schlachtviehs}

Bezüglich der Herkunft des hauptstädtischen Schlachtviehs hat man für die Spätantike die Ansicht vertreten, daß Konstantinopel aus seinem Hinterland beliefert worden ist. ${ }^{46}$ Da die Einwohnerzahl der Hauptstadt im 8. und 9. Jahrhundert ganz erheblich geringer war als im 4., 5. und 6. Jahrhundert, ${ }^{47}$ sollte man demnach davon ausgehen, daß sich diese Verhältnisse nicht geändert hatten und das Schlachtvieh aus Gegenden stammte, die höchstens 200 Kilometer entfernt lagen, also in erster Linie aus Thrakien und Bithynien.

${ }^{44}$ Zum Wildverzehr als Luxus der Oberschicht s. Kekaumenos, Strategikon 115 (48,7-8 Wassiliewsky - Jernstedt); Albert von Aachen 2,7 (304 D Bongars); A. Karpozilos - A. Kazhdan, ODB I 621 s. v. Diet; zu den exorbitanten Preisen, die in der Spätantike für Wild und Wildgeflügel zu zahlen waren, s. das Preisedikt Diokletians 4,17-20; 24; 32-33 Lauffer. Zum Wildfleisch an der kaiserlichen Tafel vgl. Theophylaktos Simokates 4,7,2 (162,5-6 DE Boor); dazu P. Schreiner, Theophylaktos Simokates. Geschichte. Stuttgart 1985, 295-296; zur Jagd als Beschäftigung des Kaisers und des kaiserlichen Hofes s. Niketas Choniates 40,61-63; 318,29-30; 450,62 van Dieten; s. zusammenfassend A. Karpozilos - J. W. Nesbitt - A. Cutler, ODB II 958 s. v. Hunting.

45 S. A. Kazhdan - J.W. NesbitT, ODB II 801-802 s. v. Fowl, domestic.

${ }^{46} \mathrm{~S}$. Herz, Lebensmittelversorgung $304,328$.

${ }^{47}$ S. C. Mango, Le développement urbain de Constantinople ( $\mathrm{IV}^{\mathrm{e}}-\mathrm{VII}^{\mathrm{e}}$ siècles). Paris 1990 , 53-54; zur Diskussion um die Einwohnerzahl s. auch Mǘler, Getreide passim, bes. $17-20$. 
Einen Hinweis auf die Herkunft des in Konstantinopel auf den Markt gelangten Schlachtviehs liefert die Bestimmung 15,3 des Eparchenbuches, in der den $\mu \alpha x \varepsilon \lambda \alpha$ óo gestattet wird, bereits jenseits des Sagaros (Sangarios) mit auswärtigen Viehzüchtern zusammenzutreffen, die ihre Herden heran-

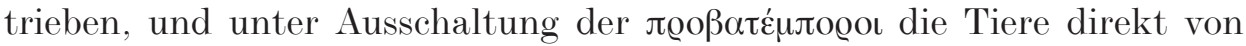
ihnen zu erwerben. ${ }^{48}$ Diese Anordnung belegt, daß Konstantinopel eben nicht allein aus seinem Hinterland mit Schlachttieren versorgt wurde, sondern daß ein Fernhandel existierte, bei dem aus dem inneren Kleinasien Viehherden, in erster Linie wohl Rinder und auch Schafe, ${ }^{49}$ von den Produzenten selbst nach der Hauptstadt getrieben wurden. Nähere Angaben über die Herkunftsregionen des Viehs macht das Eparchenbuch nicht, doch wissen wir aus anderen Quellen, daß besonders die Landschaft um Dorylaion im nordwestlichen Phrygien für ihre fetten Weidegründe berühmt war, ${ }^{50}$ was durchaus zu den Angaben des Eparchenbuches paßt. ${ }^{51}$ Die aus dem inneren Kleinasien herangetriebenen Herden wurden zunächst nach Nikomedia geführt, wo sich ein großer Viehmarkt befand, der von den hauptstädtischen Aufkäufern frequentiert wurde.$^{52}$ Von dort gelangten die Tiere - wahrscheinlich primär zu Schiff ${ }^{53}$ - nach Konstantinopel. Ein weiterer großer Viehmarkt scheint sich in der kleinen Hafenstadt Pylai (ca. $40 \mathrm{~km}$ westlich von Nikomedia) befunden zu haben, wo sich gleichfalls zahlreiche Viehherden sammelten, um in die Hauptstadt gebracht zu werden $;{ }^{54}$ die Lage der Stadt läßt die Vermutung zu, daß das Vieh in Pylai hauptsächlich aus Nordwestkleinasien, jedenfalls aus Gebieten westlich des Sangarios stammte.

Wie Quellen aus dem 12. Jahrhundert nahelegen, wurde der hauptstädtische Viehmarkt damals nicht nur aus Kleinasien, sondern in hohem Maße

${ }^{48}$ Eparchenbuch 15,3 (616-620 KoDER). Der Interpretation dieser Stelle durch Herz, Lebensmittelversorgung 327 und A.143, der die Erlaubnis für die $\mu \alpha x \varepsilon \lambda \dot{\alpha} \varrho\llcorner o เ, a u ß e r h a l b$ Konstantinopels Vieh zu erwerben, bestreitet, dürfte ein Übersetzungsfehler zugrunde liegen.

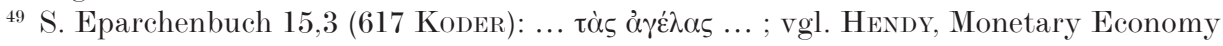
564.

${ }^{50}$ S. bes. Johannes Kinnamos 294,12-19; 295,5-6 Меineke.

${ }^{51}$ Dorylaion lag ca. 30 km südlich des Sangarios; die Stadt war überdies ein Verkehrsknotenpunkt, da sich hier die Fernstraßen ins innere und südliche Kleinasien kreuzten.

${ }^{52}$ S. Eparchenbuch 15,3 (617-618 KODER).

${ }_{53}$ Vgl. Odo von Deuil, De profectione Ludovici VII in orientem 88,10-12 BERRY; Leon von

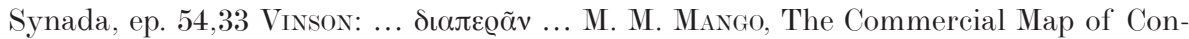
stantinople. DOP 54 (2000) 199-200 schließt aus den Patria Konstantinupoleos 2,46a (175,11-19 Preger), daß Schweine im Julianshafen angelandet und von dort zum Tauros getrieben wurden; Schafe wurden ihr zufolge im Prosphorion ausgeschifft.

${ }^{54}$ Leon von Synada, ep. 54,28-34 VINson. 
aus den europäischen Reichsgebieten beliefert. ${ }^{55}$ Auch hier ist anzunehmen, daß die Herkunftsregionen des Viehs nicht allein im Umland Konstantinopels zu suchen sind, sondern weiter nordwestlich, im Süden des Balkangebirges, namentlich im Tal der Maritza um Philippopolis und in der Gegend von Berrhoia. ${ }^{56}$ Daß das Eparchenbuch diese Gebiete nicht erwähnt, hat m. E. seinen Grund in der außenpolitischen Lage zum Zeitpunkt seiner Abfassung (ca. 912). ${ }^{57}$ Damals nämlich gehörten diese Gebiete nicht zum byzantinischen Staat, sie waren rund 100 Jahre früher an die Bulgaren verlorengegangen, ${ }^{58}$ selbst die Handelsbeziehungen waren seit dem erneuten Aufflammen der Feindseligkeiten im Jahre 894 unterbrochen. ${ }^{59}$ Ihre große Bedeutung für die hauptstädtische Fleischversorgung haben die europäischen Gebiete wohl erst nach der endgültigen Niederwerfung Bulgariens 1018 und verstärkt nach dem Verlust Zentralkleinasiens in den siebziger Jahren des 11. Jahrhunderts erlangt. ${ }^{60}$

In wieweit sich der Verlust Zentralkleinasiens seit 1073 auf die Herkunft des nach Konstantinopel getriebenen Schlachtviehs und auf die Fleischversorgung generell auswirkte, läßt sich kaum beurteilen. Es deutet einiges darauf hin, daß die Lieferungen von Schlachtvieh aus den europäischen Reichsteilen und später aus Westkleinasien eine mehr als ausreichende Kompensation für die verlorenen Zufuhrgebiete ermöglichten. ${ }^{61}$ Man muß aber in Rechnung stellen, daß vor allem die Schaf- und Rinderhändler die Möglichkeit wahrnahmen, ihr Vieh von den türkischen Nomaden zu erwerben, die seit dem ausgehenden 11. Jahrhundert das Innere Kleinasiens mit ihren Herden durchzogen; ${ }^{62}$ in diesem Falle hätten die territorialen

55 Zur Bedeutung Thrakiens speziell für die Rindfleischversorgung vgl. Johannes Kinnamos 299,16-17 Meineke.

56 Zum Viehreichtum dieser Gebiete s. Niketas Choniates 372,46-49 van DieTEN; speziell zum Überfluß Berrhoias an Rindern und Schafen s. die anonyme Historia de expeditione Friderici 44,26-28 Chroust; Villehardouin 444-445 (II 258,16-260,2 FARAL), 492 (II 306,3-10 FARAL); die reichen Weidegründe erwähnt auch die Kölner Königschronik 145,31-32 WAITZ.

57 Zur Abfassungszeit s. KoDER, Eparchenbuch 31-32.

58 S. G. Ostrogorsky, Geschichte des Byzantinischen Staates. München 1963, 169 und die Karte 219.

59 Vgl. Johannes Skylitzes 175,75-176,88 Thurs; dazu Ostrogorsky, Geschichte 212-213; das Eparchenbuch 9,6 (439-440 KODER) nennt allerdings Kontakte zu bulgarischen Leinen- und Honighändlern.

${ }^{60} \mathrm{Zu}$ byzantinischen Bemühungen, den Verlust der kleinasiatischen Agrargebiete auszugleichen, vgl. allgemein Koder, Gemüse 107; M. Angold, The Byzantine Empire 1025-1204. A Political History. London 1984, 253; 256-258.

${ }_{61}$ S. die unter A. 30 aufgeführten Quellen.

62 S. Johannes Kinnamos 295,5-6 Meineke; Niketas Choniates 176,60-61 van Dieten; P. Magdalino, The Empire of Manuel Komnenos 1143-1180. Cambridge 1993, 124. 
Einbußen letztlich keine gravierenden langfristigen Folgen für den Fleischmarkt gehabt.

Man kann also nicht sagen, daß die Versorgung Konstantinopels mit Schlachtvieh allein aus seinem Hinterland erfolgte. Vielmehr wurden dazu Gebiete herangezogen, die $400 \mathrm{~km}$ und mehr von der Hauptstadt entfernt lagen. Der von mehreren Quellen bezeugte Viehreichtum der näheren Umgebung Konstantinopels ${ }^{63}$ darf nicht auf die dort betriebene Viehzucht zurückgeführt werden, sondern steht mit bestimmten Eigentümlichkeiten des Schlachtviehfernhandels in Zusammenhang, die im folgenden näher erläutert werden sollen.

\section{Der Schlachtviehfernhandel und seine Organisation}

Die Schlachtung und der Verkauf an den Verbraucher stellten das letzte Stadium eines durchaus komplexen Prozesses dar, der mit der Aufzucht des Schlachtviehs - in, wie wir gesehen haben, räumlich oft weit entfernten Gebieten - begann und zumindest den Transport in die Hauptstadt und den Handel auf den dortigen Viehmärkten einschloß. Wie das Eparchenbuch bezeugt, hat es Züchter gegeben, die ihre Tiere selbst auf den Markt trieben und an die Metzger verkauften, eine Verfahrensweise, die auch von der kaiserlichen Regierung bevorzugt wurde ${ }^{64}$ Daneben gab es aber auch spezialisierte Händler, die das Vieh bei den Züchtern direkt oder auf lokalen Viehmärkten erwarben bzw. durch ihre Geschäftsagenten aufkaufen ließen, in eigener Regie gen Konstantinopel trieben und dort weiterverkauften. ${ }^{65}$ Die Tätigkeit dieser Zwischenhändler wurde vom Staat mehr geduldet als gefördert. Eine Untersuchung der Modalitäten des Viehhandels und der damit verbundenen Geschäftsrisiken wird zeigen, warum sich dennoch ein spezialisierter Schlachtviehhandel entwickeln und behaupten konnte.

Der Aufbau einer Viehagentur, der Ankauf der Herden und ihr Transport zu den Fleischmärkten erforderten vom Viehhändler zunächst einmal hohe Investitionen. ${ }^{66}$ Nicht nur der Einkaufspreis für die Herden, sondern auch die Lohnkosten für die Einkäufer und die Treiber, die Transport-

${ }^{63}$ Zum Überfluß an Schlachtvieh in der Umgebung Konstantinopels s. Leon von Synada ep. 54,30-32 Vinson; für das Jahr 1203 vgl. auch Villehardouin 131 (I 132,12-15 FARAL).

${ }^{64}$ Eparchenbuch 15,3-15,4 (616-623 Koder); 16,3 (640-642 Koder).

${ }^{65}$ Eparchenbuch 15,4 (621-622 Koder).

${ }^{66}$ Zur Kapitalintensität als Merkmal des Viehfernhandels s. Pickel, Viehhandel 43; 55. 
kosten - für die Fütterung unterwegs ${ }^{67}$ und die Schiffspassage nach Konstantinopel - schlugen hier zu Buche. Hinzu kam noch ein weiterer kostenträchtiger Faktor. Durch die Strapazen eines oft wochenlang währenden Triebes waren die Tiere naturgemäß in ihrem Fleischertrag und in der Fleischqualität gemindert. Für den Viehhändler bzw. für den Selbstvermarkter oder Metzger ergab sich deshalb die Notwendigkeit, dem Vieh vor dem Endverkauf oder der Schlachtung eine gewisse Zeit der Erholung zu gönnen und es durch ausreichende Fütterung wieder Fleisch ansetzen zu lassen, wollte er die größtmögliche Einnahme erzielen ${ }^{68}$ Eine solche Endmast ergab natürlich nur dann einen Sinn, wenn sie in unmittelbarer Nähe des Viehmarktes bzw. des Schlachthofes stattfand, oder aber an einem Ort, von dem der Weitertransport nurmehr per Schiff vonstattenging. Das erforderte Weideplätze oder Pferche mit ausreichendem Futtervorrat in der unmittelbaren Umgebung der Hauptstadt, die gekauft oder von den Besitzern gemietet werden mußten. Das Verfahren, in Stadtnähe umfangreiche Weideflächen zu unterhalten, auf denen das Vieh bis zur Schlachtung gemästet wurde, ist aus Westeuropa vielfach bezeugt: ${ }^{69}$ Hinweise darauf, daß dies auch im byzantinischen Machtbereich so gehandhabt wurde, finden sich zur Genüge.$^{70}$ Für Konstantinopel selbst sind große unbebaute Areale innerhalb der Stadtmauern bezeugt - laut Odo von Deuil, der die Stadt im Jahre 1147 besuchte, lag ein Drittel der ummauerten Fläche brach - und diese wurden vielfach landwirtschaftlich genutzt. ${ }^{71}$ An Weideflächen und

${ }^{67} \mathrm{Zu}$ den Kosten für den Unterhalt der Tiere auf dem Trieb vgl. W. V. Stromer, Zur Organisation des transkontinentalen Ochsen- und Textilhandels im Spätmittelalter. Der Ochsenhandel des Reichserbkämmerers Konrad von Weinsberg anno 1422, in: Internationaler Ochsenhandel 184.

${ }^{68}$ Zur Festlegung der Preise durch den Eparchen und zur Rolle des Gewichts s. u.; zur Beurteilung der Fleischqualität der geschlachteten Tiere, die ebenfalls unter der direkten Aufsicht des Eparchen erfolgen sollte, s. Eparchenbuch 15,2 (611-613 KoDER).

${ }^{69}$ S. F. Lerner, Die Bedeutung des internationalen Ochsenhandels für die Fleischversorgung deutscher Städte im Spätmittelalter und der frühen Neuzeit, in: Internationaler Ochsenhandel 198-199; 212; F. InsigLer, Zum Kölner Viehhandel und Viehmarkt im Spätmittelalter, ibid. 226; zum spätantiken Rom s. Herz, Lebensmittelversorgung 290.

${ }^{70}$ Weiden in unmittelbarer Stadtnähe, auf denen das Vieh im Belagerungsfall zumindest eine Zeitlang ernährt und zugleich vor feindlichem Zugriff geschützt werden konnte, erwähnt Prok. II 26,5 (I 269,3-11 HaURY - WIRTH); vgl. auch die Historia de expeditione Friderici 44,26-30 Chroust, die explizit überliefert, daß sich die deutschen Kreuzfahrer erst nach der Eroberung Berrhoias der umfangreichen Herden bemächtigen konnten. Große Weideflächen in Stadtnähe existierten auch im Falle Belgrads und Nikaias, s. Albert von Aachen I 6; 16 (275 C; 284 D Bongars).

${ }^{71}$ Odo von Deuil, De profectione Ludowici VII in orientem 64,11-12 BERRY: Latus tertium de trigono civitatis campos habet ...; vgl. 15-16 Berry. Koder, Gemüse 70 (s. auch ders., 
Platz für Viehkorräle kann es also in der Hauptstadt nicht gemangelt haben.

Trotz des hohen Kapitalbedarfs war der spezialisierte, in Konstantinopel ansässige Viehhändler gegenüber dem auswärtigen Selbstvermarkter in mehrfacher Hinsicht im Vorteil. Zum einen dürfte es für ihn leichter gewesen sein, mit hauptstadtnahen Landbesitzern und Schiffseignern günstige Kontrakte zu schließen, um die Schlußmast und den Transport zu den Märkten zu ermöglichen; zum anderen konnte er durch Zusammenstellung von Herden aus besonders hochwertigen Tieren einen qualitativen Vorsprung und dadurch einen entsprechenden finanziellen Gewinn erzielen. ${ }^{72}$ Außerdem besaßen die hauptstädtischen Viehhändler einen besseren Überblick über das Marktgeschehen als die aus der Ferne angereisten Züchter, konnten also ihre Ein- und Verkäufe längerfristig planen, Vieh während etwaiger Niedrigpreisperioden auf den eigenen oder gepachteten Weiden zurückhalten und dadurch ihre Gewinne weiter steigern. Der Selbstvermarkter lief viel eher Gefahr, bei einem Überangebot auf seinem Vieh sitzen zu bleiben und unter Preis verkaufen zu müssen, zumal ein Weiterverkauf an die Zwischenhändler ausdrücklich untersagt war. ${ }^{73}$ Ein Produzent, der selbst in Konstantinopel ansässig war und das Vieh von seinen eigenen Gütern durch seine eigenen Leute in die Hauptstadt bringen ließ, hatte natürlich die Möglichkeit, an Ort und Stelle die notwendigen logistischen Strukturen aufzubauen. Dieser Typ scheint aber nicht verbreitet gewesen zu sein ${ }^{74}$ sodaß er niemals zu einer ernsthaften Konkurrenz für die selbständigen Schlachtviehhändler wurde.

Fresh vegetables 53) schätzt die landwirtschaftlich nutzbaren Brachflächen innerhalb der Mauern auf 2-3 km²; hinzu wären noch wenigstens $10 \mathrm{~km}^{2}$ vor den Stadtmauern zu rechnen.

${ }^{72}$ Ein derartiges Verfahren versprach reiche Gewinne, vgl. InsigLer, Kölner Viehhandel 223 zum Konflikt der Kölner mit den Neusser Viehhändlern, die aus den von Norden gen Köln getriebenen Herden die besten Tiere aufzukaufen versuchten, um sie anschlieBend selbst in Köln auf den Markt zu bringen.

${ }^{73}$ S. Eparchenbuch 16,3 (640-643 Koder). Diese Anordnung bezieht sich auf die Schweinevermarktung am Tauros, doch fügt sie sich gut in den allgemeinen Kontext kaiserlicher Maßnahmen zur Begrenzung des preistreibenden Zwischenhandels ein, sodaß man sie m. E. in Analogie auch auf den Schaf- und Rindermarkt beziehen kann.

${ }^{74}$ Kapitalintensive Handelsgeschäfte byzantinischer Grundherrn kamen vor, wie aus dem Strategikon des Kekaumenos (114 [47,32-48,2 WAssILIEWsky - JERnstedT]; 115 [48,1016 Wassiliewsk - JERNstedT]) hervorgeht, wurden aber, wie die gleichen Stellen deutlich machen, von Standesgenossen mit Mißtrauen und auch mit spürbarem Degout betrachtet. 


\section{Die hauptstädtischen Fleischmärkte}

Konstantinopel besaß keinen zentralen Schlachtviehmarkt. Schweine und Milchlämmer wurden am Tauros, ${ }^{75}$ Schafe am Strategion verhandelt und geschlachtet; ${ }^{76}$ wo Rinder und Rindfleisch angeboten wurden, ist nicht bekannt. Unklar bleibt auch, ob die Schlachtung in speziellen Gebäuden oder unter freiem Himmel stattfand. ${ }^{77}$

Einige Probleme werfen die Angaben auf, die das Eparchenbuch über die Modalitäten des Viehhandels auf den hauptstädtischen Märkten macht. Der Eparch bzw. dessen Beauftragte nahmen eine Zählung der angebotenen Tiere vor und legten die Preise fest ${ }^{78}$ wobei bei Schafen das Nomisma als

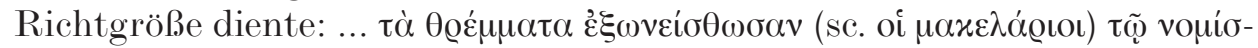

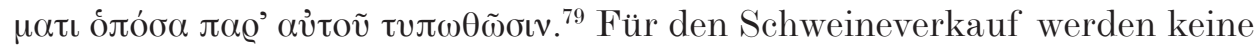
derartigen Bestimmungen überliefert, doch wird man sie in Analogie zum Schafhandel ohne weiteres voraussetzen können. Sicher ist also, daß das Angebot den behördlich festgelegten Preis beeinflußte, ${ }^{80}$ der Text legt ferner nahe, daß für ein Nomisma normalerweise mehrere Tiere erworben werden konnten. Bezogen auf Schafe und Schweine erscheint das als einsichtig: Preisangaben aus dem frühen 8. Jahrhundert zeigen, daß damals in Ägypten der Preis für ein Schaf ein halbes Nomisma oder geringfügig weniger betrug, für Schweine lag er etwas höher (1 bzw. 2/3 Nomisma pro Stück) ${ }^{81}$ wobei berücksichtigt werden muß, daß Schweine in Ägypten sehr wahrscheinlich teurer waren als in Konstantinopel. ${ }^{82}$ Wie aber verhielt es sich mit den wesentlich schwereren Rindern? Selbst wenn man die höhere Qualität von Schaf- und Schweinefleisch in Rechnung stellt, wird man

75 Eparchenbuch 15,5 (525-526 Koder); 16,2 (635-636 Koder); 16,3 (642 Koder).

76 Eparchenbuch 15,1 (608-610 KodER); 15,5 (624-625 KodER).

77 M. M. Mango, Commercial Map 199-200 vermutet, daß die Schlachtung nicht im Freien, sondern in an die Märkte angrenzenden Gebäuden erfolgte; in diesem Falle würde sich die Frage nach der Gebäudemiete stellen (vgl. z.B. Eparchenbuch 18,5 [690 KoDER]).

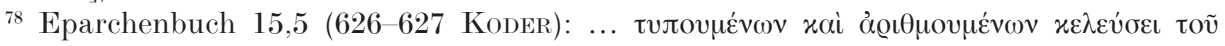

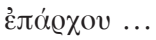

79 Eparchenbuch 15,1 (609-610 KODER).

80 Analoge Verhältnisse lassen sich beim Handel mit frischem Fisch, Brot und Wein nachweisen, s. Eparchenbuch 17,4 (667-670 KodEr), 18,4 (686-688 KoDER) und 19,1 (697703 KODER).

81 S. P. Lond. 1414,6. 37. 66; 1448,13-14. 33-34; die Angaben erfolgen unter der Prämis-

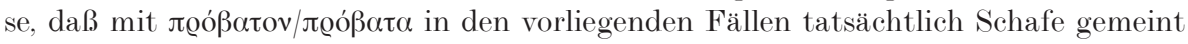
sind; der geringe Unterschied zu den Schweinepreisen legt dies jedoch nahe, vgl. schon Ostrogorsky, Löhne und Preise 332.

82 Der Mangel an ausgedehnten Wäldern schlug hier sicherlich zu Buche, vgl. dazu o. 
schon aufgrund des bei Rindern erheblich größeren Fleischertrages und der besseren Verwertbarkeit dieses Tieres von einem Preis ausgehen können, der ein mehrfaches desjenigen für Schafe und Schweine betrug. Hier liegt ein eindeutiger Hinweis darauf vor, daß der Handel mit Rindern und

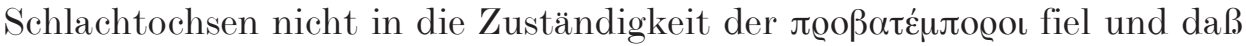
für die Vermarktung von Rind- und Kalbfleisch eigene, im Eparchenbuch nicht genannte Korporationen existierten.

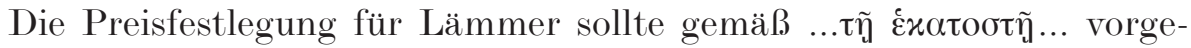
nommen werden, nach dem Hundertstel also. ${ }^{83}$ Daß damit der hundertste Teil eines Nomisma gemeint ist, wie es der Text an sich nahelegt, ist bezweifelt worden, man nahm an, daß in Wirklichkeit das Miliaresion, also der zwölfte Teil eines Nomisma als Maßstab diente. ${ }^{84}$ Das Gewichtsverhältnis zwischen einem Schaf und einem Lamm spricht jedenfalls für die Richtigkeit dieser These.

In diesem Zusammenhang stellt sich generell die Frage, wie sich Gewicht und Qualität der Tiere auf den Preis auswirkten. Aus den Formulierungen des Eparchenbuches könnte man den Schluß ziehen, daß diese Aspekte keine Rolle spielten und es ganz der Geschäftstüchtigkeit des Metzgers überlassen blieb, pro Nomisma besonders hochwertige Tiere einzukaufen. Andererseits wird im Kapitel über den Schweinehandel erwähnt,

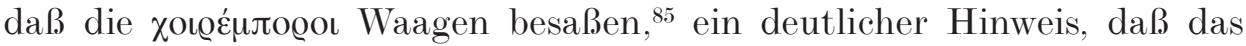
Gewicht beim Verkauf berücksichtigt wurde; man kann dies m. E. auch für den Schaf- und Rinderhandel voraussetzen. Als plausibel erscheint die Vermutung, daß die Preisfestlegung allein nach dem zahlenmäßigen Angebot erfolgte und daß das Wiegen des Schlachtviehs nur bei den Verhandlungen zwischen Viehhändlern und Metzgern ins Spiel kam, wenn letztere ihre Kaufentscheidung vom Gewicht der Tiere abhängig machten. ${ }^{86}$ Dafür spricht m. E. noch eine weitere Beobachtung: bei den seitens des Eparchen festgesetzten Preisen handelte es sich um Höchstpreise, der Verkauf zu niedrigeren Preisen, d.h. von mehr Tieren pro Nomisma, war somit jederzeit möglich. ${ }^{87}$

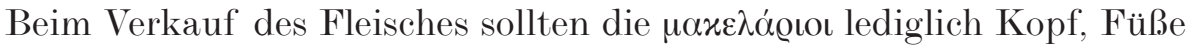
und Innereien als ihren Verdienst berechnen, das übrige Fleisch aber gemäß

${ }^{83}$ Eparchenbuch 15,5 (627-628 KoDER).

${ }^{84}$ S. Koder, Eparchenbuch 125; zum Miliaresion vgl. die Scholie zu Eparchenbuch 1,4 (app. crit. zu 58 Koder); Hendy, Monetary Economy (A. 3) 504-505.

${ }^{85}$ Eparchenbuch 16,6 (649-650 Koder).

${ }^{86}$ Vgl. CIL VI 1770,4-9; Herz, Lebensmittelversorgung 283.

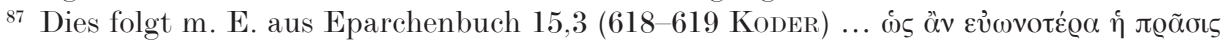

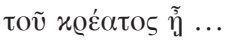


dem Ankaufspreis verkaufen. ${ }^{88}$ Diese Bestimmung befremdet auf den ersten Blick. Zwar waren auch die Gewinnsätze anderer Berufsgruppen staatlich begrenzt, doch wurden die Spannen stets in Geld angegeben, sie betrugen in der Regel ein Miliaresion pro Nomisma, also maximal ca. $8 \%{ }^{89}$ Im vorliegenden Fall aber sollte der Metzger seinen Gewinn aus dem Verkauf der Innereien und einiger minderwertiger, weil nicht sehr fleischhaltiger Partien erzielen. Vor dem Hintergrund der nahezu vollständigen Verwertbarkeit des Schlachtviehs wird jedoch sehr schnell deutlich, daß die Gewinnmöglichkeiten des Metzgers nicht so eng begrenzt waren, wie es zunächst den Anschein hat. Zum Kopf gehörte bei Rindern, Widdern und Ziegen auch das Horn, das bestimmten Berufsgruppen (z.B. den Kammachern; Bogenbauern; Rüstungsherstellern) als Rohmaterial diente; ${ }^{90}$ bei den Innereien ließen sich nicht bloß die für den menschlichen Verzehr geeigneten Teile (Leber, Nieren, Herz, Lunge etc.) absetzen, sondern auch die Därme samt Darminhalt, der als Dünger verwendet wurde, ${ }^{91}$ und der Talg, der bei Lichtziehern und Seifensiedern Abnehmer fand. ${ }^{92}$ Man kann daher durchaus annehmen, daß sich die bei Ausnutzung aller Möglichkeiten erzielbare Gewinnspanne der $\mu \alpha x \varepsilon \lambda \alpha ́ \varrho ı$ durchaus im Rahmen dessen bewegte, was anderen Berufsgruppen als Maximum zugestanden wurde. ${ }^{93}$ Leider macht das Eparchenbuch keine Angaben darüber, nach welchem Maßstab die Schweineschlächter ihre Gewinne berechnen sollten; man darf vermuten, daß man auch ihnen die übliche Nettogewinnspanne von rund $8 \%$ einräumte, ohne daß wir wüßten, auf welche Weise diese erzielt werden sollte.

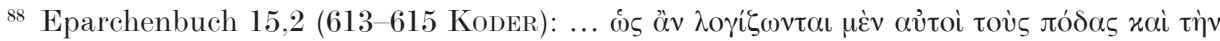

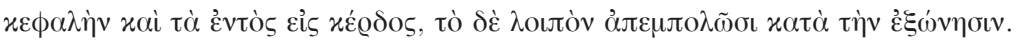

${ }^{89}$ Koder, Eparchenbuch 35 mit Literatur.

${ }_{90}$ Zum Bogenbau und den dazu benötigten Rohmaterialien s. T. Kolias, Byzantinische Waffen. Ein Beitrag zur byzantinischen Waffenkunde von den Anfängen bis zur lateinischen Eroberung. Wien 1988, 214-216; auch Tiersehnen, besonders von Rindern, fanden dabei Verwendung; zu Panzern aus Hornplättchen s. Kolias 45 und A. 65; 54.

${ }^{91}$ S. Stromer, Ochsenhandel 183.

${ }_{92}$ S. Eparchenbuch 11,4 (510-512 Koder); 12,8 (553-555 Koder).

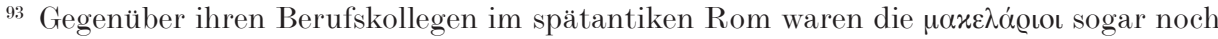
besser gestellt. Eine um 363 erlassene Verordnung des praefectus urbi gestand dem Metzger nur Kopf, Füße und Fett zu, während der Käufer neben dem Fleisch auch Fell und Innereien erhalten sollte, s. CIL VI 1770,9-12. Allerdings hat man hier zu berücksichtigen, daß die Viehmetzger in viel stärkerem Maße auch Viehhändler waren und es sich bei ihrem Vieh zum Teil um Steuertiere gehandelt haben kann, s. Herz, Lebensmittelversorgung 283. 
Allerdings wird im Eparchenbuch den Metzgern ausdrücklich gestattet, sich selbst am Fernhandel zu beteiligen. ${ }^{94}$ Entscheidend war dabei, daß die Festsetzung der Preise durch den Eparchen erst am Strategion und am Tauros anhand des angebotenen Viehs geschah. Wenn der im Fernhandel tätige Metzger das Fleisch seiner zu einem niedrigeren als dem festgesetzten Tagespreis angekauften Tiere auf den Markt brachte, konnte er dadurch die staatlich erlaubte Gewinnspanne erhöhen.

Neben den Märkten für Frischfleisch gab es in Konstantinopel auch einen Handel mit konserviertem Fleisch, für den jedoch eine spezielle Korporation, die der sogenannten $\sigma \alpha \lambda \delta \alpha \mu \alpha ́$ oı (Gemischtwarenhändler) zuständig war, deren Angebot neben Gebrauchswaren und anderen Lebensmitteln auch gepökeltes oder geräuchertes Fleisch umfaßte. ${ }^{95}$ Wo diese ihre Ware erwarben, ob sie sich bei den Metzgern oder den Vieh- und Schweinemetzgern eindeckten und das Fleisch dann selbst konservierten, ist nicht überliefert. Wir besitzen lediglich aus dem späten 5. Jahrhundert einen Beleg dafür, daß Salzfleisch per Schiff nach Konstantinopel transportiert wurde ${ }^{96}$ wie überhaupt ein Fernhandel mit konserviertem Fleisch schon für die Antike belegt ist. ${ }^{97}$ Wir können daher davon ausgehen, daß die Gemischtwarenhändler das Fleisch in der Regel bereits im konservierten Zustand ankauften; ein Kauf von Schlachttieren zwecks Konservierung und anschließendem Weiterverkauf barg ohnehin die Gefahr künstlicher Verknappung in sich und war keinesfalls im kaiserlichen Interesse. ${ }^{98}$ Bei dem Pökel- bzw. Räucherfleisch dürfte es sich vorzugsweise um Schweinefleisch gehandelt haben. ${ }^{99}$

Weder bei den die Metzger noch bei den die Gemischtwarenhändler betreffenden Verordnungen wird der Verkauf von Würsten erwähnt. Wenn man Würste als eine Form der Fleischkonserve ansieht, so läßt sich immerhin vermuten, daß der Wurstwarenverkauf in die Zuständigkeit der $\sigma \alpha \lambda \delta \alpha \mu \alpha \dot{\varrho} \iota \iota$ fiel. ${ }^{100}$ Allerdings erwähnt Prokop im 6. Jahrhundert spezielle

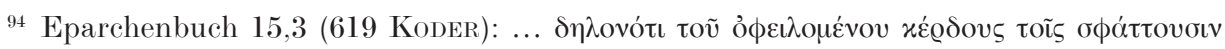

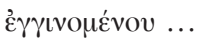

95 Eparchenbuch 13,1 (563; 568 KODER).

96 Orientis Graeci Inscriptiones Selectae 521,24; zu der Inschrift s. J. Durliat - A. Guiltou, Le tarif d'Abydos (vers 492). BCH 108 (1984) 581-598.

97 S. Das Hermipposfragment bei Athenaios I 27 e (I 62,12 KAIBEL); Varro, Rerum rusticarum 2,4,10 (230,2-4 KEILL).

$98 \mathrm{Vgl}$. Eparchenbuch 16,5 (646-648 Koder); s. dazu u.

99 Vgl. Eparchenbuch 15,6 (629-630 KodER); 16,5 (646-647 KodER); Varro, Rerum rusticarum 2,4,10 (230,2-4 KEILL).

$100 \mathrm{Vgl}$. Eparchenbuch 13,1 (563 Koder). 
Wurstverkäufer in Daras, ${ }^{101}$ betreffs Konstantinopel fehlen jedoch Belege für die Existenz eines solchen Gewerbes. Dies kann bedeuten, daß die Wurstverkäufer bei der Redaktion des Eparchenbuches übergangen wurden, was vielleicht darin begründet liegt, daß die Herstellung und der Verkauf von Würsten kein bedeutender Geschäftszweig war. ${ }^{102}$

\section{Staat und Fleischmarkt}

Im Mittelpunkt des kaiserlichen Interesses am Fleischmarkt stand die Sicherung niedriger Fleischpreise. Diesem Ziel diente zunächst die staatliche Preiskontrolle, die, wie wir gesehen haben, den Verkauf des Schlachtviehs und der geschlachteten Tiere unter behördliche Aufsicht stellte und dem Preiswucher durch Festlegung von Höchstpreisen entgegenzuwirken versuchte. Vor allem war man kaiserlicherseits bemüht, den Schlachtviehzwischenhandel zu beaufsichtigen und durch strenge Auflagen nach Möglichkeit einzuschränken; nicht weniger als sieben der insgesamt zwölf Verordnungen, die den Frischfleischmarkt betreffen, sind diesen Anliegen gewidmet. ${ }^{103}$ Ursache des Mißtrauens gegenüber dem Zwischenhandel war die Furcht vor einer durch die Händler selbst herbeigeführten künstlichen Verknappung des Angebots zwecks Erzielung höherer Gewinne. ${ }^{104}$ Zweifellos war diese Furcht nicht unbegründet. Die oben dargelegten Modalitäten des Schlachtviehfernhandels und hier vor allem die Benutzung hauptstadtnaher Weiden und Mastplätze luden zur Manipulation geradezu ein; das Verbot, Selbstvermarkter daran zu hindern, ihre Tiere nach Konstantinopel zu treiben, ${ }^{105}$ zeigt, daß die Schlachtviehhändler auch vor unlauteren Methoden nicht zurückschreckten, um die Marktlage zu ihren Gunsten zu beeinflussen. Eine weitere Verordnung verpflichtete die Obmänner der

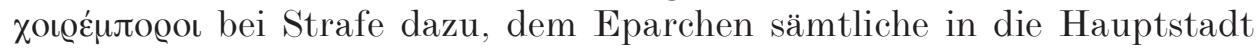
getriebenen Schweineherden anzuzeigen und dafür Sorge zu tragen, daß diese nicht an ihre Berufsgenossen veräußert wurden; ${ }^{106}$ von der Existenz

\footnotetext{
101 Prok. I 26,9 (I 143,17 Haury - WirTh).

102 Ausweislich des diokletianischen Preisediktes (4,6-7; 15 Lauffer) gehörten Wurstwaren, wie konserviertes Fleisch überhaupt, zu den teureren Nahrungsmitteln; es dürfte freilich mit einer begrenzten Selbstversorgung durch Hausschlachtung zu rechnen sein.

${ }^{103}$ Eparchenbuch 15,3; 15,4; 15,5 (616-628 KODER); 16,2; 16,3, 16,4 (635-645 KodER); 16,6 (649-651 KODER).

104 S. besonders Eparchenbuch 15,3 (618-620 Koder); 16,3 (640-643 KodER); 16,4 (644-645 KODER).

${ }^{105}$ Eparchenbuch 15,4 (622-623 KodER).

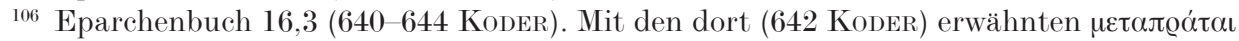

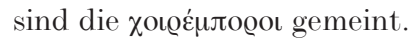


analoger, den Schaf- und Rinderhandel betreffenden Anordnungen ist auszugehen. ${ }^{107}$

Neben der Sicherstellung einer ungehinderten Selbstvermarktung versuchte die kaiserliche Regierung auch, die Metzger dazu zu ermuntern, sich unter Umgehung des Zwischenhandels außerhalb der hauptstädtischen Märkte selbst mit den Züchtern zu treffen und das Vieh von ihnen preisgünstig zu erwerben. Zwar betrifft die überlieferte Bestimmung nur die

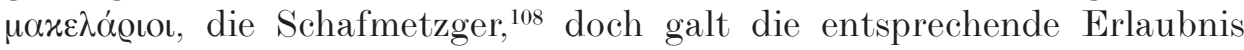
zweifellos auch für die Schweine- und Rinderschlächter. ${ }^{109}$ Wollte ein Metzger von dieser Erlaubnis Gebrauch machen, so bedingte das freilich, daß er zumindest einen erklecklichen Teil der Transportkosten und des Transportrisikos zu übernehmen hatte, von den Kosten für die empfehlenswerte Endmast einmal abgesehen. Ob viele Metzger außer dem notwendigen Kapital auch die Risikobereitschaft aufbrachten, um neben ihrem Handwerk auch im Schlachtviehfernhandel tätig zu sein, scheint zweifelhaft. Die kaiserlicherseits verfügte Beschränkung ihrer Gewinne erschwerte ihnen nämlich die notwendige Kapitalbildung, und von irgendwelchen staatlichen Förderprogrammen zugunsten der Metzger - etwa durch Gewährung von Steuererleichterungen oder billigen Darlehen zum Aufbau einer Viehagentur - ist nichts bekannt. ${ }^{110}$ Das verständliche kaiserliche Bestreben, durch Preiskontrollen und Gewinnbeschränkungen dem Preiswucher auf den Fleischmärkten entgegenzuwirken, begünstigte in gewisser Weise die ungeliebten, aber kapitalkräftigeren Schlachtviehzwischenhändler.

Man darf generell bezweifeln, ob die staatlichen Anstrengungen, den Zwischenhandel zu kontrollieren oder gar einzuschränken, von großem

${ }^{107}$ Von derartigen Bestimmungen waren auch die Fischhändler und Kneipenwirte betroffen, deren Obmänner dem Eparchen über die Menge der gefangenen Fische bzw. die Anlieferung des Weins Meldung erstatten mußten, s. Eparchenbuch 17,4 (667-671 KoDER), 19,1 (697-701 KODER).

108 Eparchenbuch 15,3 (616-620 Koder).

109 Die die Schweinemetzger betreffende Verordnung 16,2 (635-639 Koder) scheint auf den

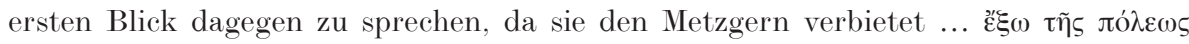

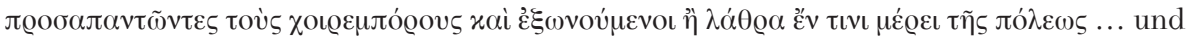
den An- und Verkauf von Schweinen auf den Tauros beschränkt. Die Erwähnung der

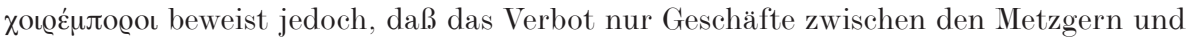
den Schweinehändlern betraf, die in Konstantinopel selbst oder der unmittelbaren Umgebung der Hauptstadt unter Ausschaltung der behördlichen Preiskontrolle getätigt wurden. Von einem Verbot für die Schweineschlächter, ihr Vieh direkt vom Züchter zu erwerben und selbst in die Hauptstadt zu bringen, ist nicht die Rede.

${ }^{110}$ Im kaiserzeitlichen Rom hatte man den Schweinehandel zeitweilig durch Steuervergünstigungen gefördert, s. HERz, Lebensmittelversorgung 162-169. 
Erfolg gekrönt waren. Das für die Schweinehändler überlieferte Verbot, Tiere im Haus eines Beamten(!) zu verstecken, ${ }^{111}$ unterstreicht letztlich die Hilflosigkeit der Regierung und liefert zugleich einen Hinweis auf das Ausmaß der auf dem Fleischmarkt obwaltenden Korruption.

Einen weiteren Schwerpunkt staatlicher Politik bildete das Bemühen, die Zuständigkeiten der einzelnen Gilden gegeneinander abzugrenzen und Übergriffe in den Geschäftsbereich anderer Korporationen unter Strafe zu stellen. ${ }^{112}$ Jede der mit der Fleischversorgung befaßten Korporationen, egal ob die der Metzger oder der Zwischenhändler, war für eine bestimmte Fleischsorte zuständig, der Handel mit anderen Sorten war ihr strikt untersagt. ${ }^{113}$ Darüber hinaus wurden die Metzger auf den Verkauf von Frischfleisch beschränkt und besaßen keine Erlaubnis, das Fleisch der von ihnen geschlachteten Tiere zu konservieren; ${ }^{114}$ der Verkauf von konservierten Fleischwaren blieb den $\sigma \alpha \lambda \delta \alpha \mu \alpha \dot{o}$ เo vorbehalten. ${ }^{115}$ Die Bestimmungen zu-

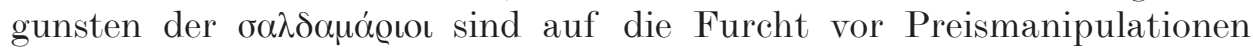
durch die Metzger zurückzuführen, ${ }^{116}$ allerdings brachten sie für diese bei der leichten Verderblichkeit des Fleisches zumal in den wärmeren Jahreszeiten die Gefahr finanzieller Verluste mit sich, wenn sie über den Tagesbedarf geschlachtet hatten. Aus heutiger Sicht schwer nachvollziehbar sind dagegen die Beschränkungen der Gilden auf jeweils eine Fleischsorte. Sie entsprachen jedoch, wie oben schon angedeutet, einer aus dem antiken Rom überlieferten Tradition; vergleichbare Regelungen sind im übrigen auch für das mittelalterliche Deutschland vereinzelt nachzuweisen. ${ }^{117}$

Was die Qualitätskontrolle und die gerade bei Schweinefleisch so wich-

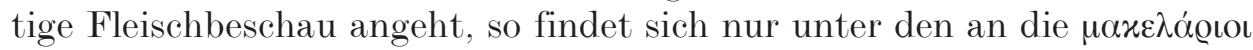
gerichteten Verordnungen ein Hinweis darauf, daß es dergleichen auf den hauptstädtischen Fleischmärkten gegeben hat. ${ }^{118}$ Demnach waren die Schafmetzger verpflichtet, das Fleisch nach der Qualität der Tiere (xat⿳亠े

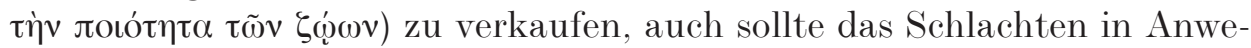
senheit des Eparchen (wohl eher seines Beauftragten) vor sich gehen. ${ }^{119}$ Für

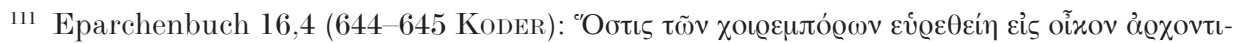

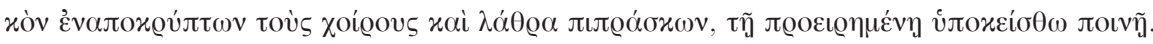

112 Diese Politik war nicht auf den Fleischmarkt beschränkt, vgl. Eparchenbuch 2,1 (157-61 Koder); 10,6 (492-493 Koder); 11,8 (521-523 Koder); 14,2 (597-606 Koder). 
den Verkauf von Schweinefleisch ist keine derartige Bestimmung überliefert, man wird sie aber $\mathrm{m}$. E. voraussetzen können.

Überhaupt nicht angesprochen wird das wichtige Problem der Abfallbeseitigung und das damit verbundene der Geruchsbelästigung, obwohl sich im Eparchenbuch ein Indiz dafür findet, daß zumindest letzterer Aspekt grundsätzlich bekannt war. ${ }^{120}$ Vermutlich blieb die entsprechende Verfügung bei der Redaktion des Eparchenbuches unberücksichtigt.

Ein Gesamturteil über die Praktikabilität der kaiserlichen Politik zur Sicherung der hauptstädtischen Fleischversorgung zu fällen ist schwierig, weil wir nichts über Teuerungen und Engpässe in der Fleischversorgung wissen. Nachrichten über Versorgungskrisen und Hungersnöte beziehen sich durchweg auf die Knappheit von Brotgetreide, was bei der vergleichsweise geringen Bedeutung, die dem Fleisch für die Ernährung zukam, nicht erstaunt. ${ }^{121}$ Daß bei Hungersnöten und Teuerungen wie denen in den siebziger Jahren des 11. Jahrhunderts auch die Fleisch- und Schlachtviehversorgung in Mitleidenschaft gezogen wurde, ist gewiß, ${ }^{122}$ es scheint jedoch, daß es von solchen Notzeiten abgesehen im Großen und Ganzen gelang, bis zur lateinischen Eroberung eine ausreichende Belieferung sicherzustellen.

Zweifellos war die Bevölkerung Konstantinopels hinsichtlich des Fleischverzehrs privilegiert, da die notwendige Infrastruktur vorhanden war, um eine Versorgung des Marktes zu gewährleisten, und der Staat bemüht war, maßvolle Fleischpreise zu garantieren. Das gleiche dürfte auch für andere verkehrsgünstig gelegene Städte mit guten Weidemöglichkeiten wie Nikomedia, Nikaia oder Dorylaion gegolten haben. Verallgemeinern darf man diese Verhältnisse freilich nicht. ${ }^{123}$

\section{Zusammenfassung}

Das Eparchenbuch berücksichtigt nur die Schaf- und Schweinefleischmärkte und die vier dort tätigen Korporationen und läßt den Rinderhandel und die Vermarktung des Rindfleischs unerwähnt, obwohl Rindfleisch für die hauptstädtische Fleischversorgung eine bedeutende Rolle gespielt hat, die diejenige des Schaffleischs vielleicht übertraf. Der Verzehr von Schwei-

120 S. Eparchenbuch 10,1 (460-461 Koder).

121 Vgl. etwa Koder, Gemüse 106-107 mit Quellen.

122 Michael Attaleiates 155,6-11 Pérez Martín schreibt ganz allgemein von einem Mangel an Lebensmitteln.

${ }^{123}$ S. bereits Patlagean, Pauvreté 41; auf den höheren Fleischkonsum der städtischen Bevölkerung weist für Westeuropa U. Dirlmeier, LexMa III 2164 s. v. Ernährung hin. 
nefleisch stand an dritter Stelle, doch kann man nach der Eroberung Bulgariens einen Anstieg des Konsums vermuten. Konstantinopel ist nicht aus seinem Umland mit Schlachtvieh versorgt worden, sondern vor allem aus Innerkleinasien, seit dem 11. Jahrhundert verstärkt auch aus dem Balkanraum. Die Versorgung lag primär in den Händen spezialisierter Fernhändler, während der Selbstvermarktung durch die Züchter eine weit geringere Bedeutung zukam. Auf den Märkten erfolgte durch den Eparchen die Preisfestlegung nach der Zahl der in die Hauptstadt getriebenen Tiere, wobei als Richtwert für Schafe und auch für Schweine das Nomisma galt; das Wiegen der Tiere kam nur bei den Kaufverhandlungen zwischen Händlern und Metzgern ins Spiel. Der den Metzgern zugestandene Maximalgewinn von etwas über $8 \%$ mußte aus dem Verkauf der Haut, des Horns, des Fetts, der Sehnen, des Darminhalts und einiger weniger wertvoller Fleischpartien erzielt werden. Das Bemühen der Kaiser galt in erster Linie der Sicherstellung moderater Preise auf den Fleischmärkten, sie standen daher dem als preistreibend beurteilten $Z$ wischenhandel mißtrauisch gegenüber, ohne diesen wirkungsvoll kontrollieren und einschränken zu können. Wie schon das Übergehen der Rindfleischversorgung zeigt, bietet das Eparchenbuch keine durchdachte Zusammenfassung der für den Vieh- und Fleischmarkt wichtigen kaiserlichen Verordnungen, sondern hinterläßt den nachhaltigen Eindruck der Zufälligkeit. 
